\title{
BMJ Open Outcomes reported in randomised controlled trials of major depressive disorder in older adults: protocol for a methodological review
}

\author{
Myanca Rodrigues (D) , ${ }^{1}$ Nitika Sanger (D) ,2 Alexander Dufort, ${ }^{2}$ Stephanie Sanger, ${ }^{3}$ \\ Balpreet Panesar (D) , ${ }^{4}$ Alessia D'Elia (D) , ${ }^{4}$ Sameer Parpia, ${ }^{5,6}$ Zainab Samaan (D) , ${ }^{2,5,7}$ \\ Lehana Thabane (1D) 5,8
}

To cite: Rodrigues $\mathrm{M}$, Sanger N, Dufort A, et al. Outcomes reported in randomised controlled trials of major depressive disorder in older adults: protocol for a methodological review. BMJ Open 2021;11:e054777. doi:10.1136/ bmjopen-2021-054777

- Prepublication history and additional supplemental material for this paper are available online. To view these files, please visit the journal online (http://dx.doi.org/10.1136/ bmjopen-2021-054777).

Received 23 June 2021 Accepted 18 October 2021

Check for updates

(c) Author(s) (or their employer(s)) 2021. Re-use permitted under CC BY-NC. No commercial re-use. See rights and permissions. Published by BMJ.

For numbered affiliations see end of article.

Correspondence to Dr Lehana Thabane; thaban!@mcmaster.ca

\section{ABSTRACT}

Introduction Major depressive disorder (MDD or depression) is prevalent among adults aged 65 years and older. The effectiveness and safety of interventions used to treat depression is often assessed through randomised controlled trials (RCTs). However, heterogeneity in the selection, measurement and reporting of outcomes in RCTs renders comparisons between trial results, interpretability and generalisability of findings challenging. There is presently no core outcome set (COS) for use in RCTs that assess interventions for older adults with MDD. We will conduct a methodological review of the literature for outcomes reported in trials for adults 65 years and older with depression to assess the heterogeneity of outcome measures.

\section{Methods and analysis RCTs evaluating}

pharmacotherapy, psychotherapy, or any other treatment intervention for older adults with MDD published in the last 10 years will be located using electronic database searches (MEDLINE, Embase, PsycINF0 and the Cochrane Central Register of Controlled Trials). Reviewers will conduct title and abstract screening, fulltext screening and data extraction of trials eligible for inclusion independently and in duplicate. Outcomes will be synthesised and mapped to core outcome-domain frameworks. We will summarise characteristics associated with trials and outcomes.

Ethics and dissemination We hope that findings from our methodological review will reduce variability in outcome selection, measurement and reporting and facilitate the development of a COS for older adults with MDD. Our review will also inform evidence synthesis efforts in identifying the best treatment practices for this clinical population. Ethics approval is not required, as this study is a literature review.

PROSPERO registration number CRD42021244753.

\section{INTRODUCTION}

Mood disorders or affective disorders are the terms given to the group of psychiatric disorders which characterise the lowering or elevation of one's mood or emotional state. ${ }^{12}$ There are many types of mood disorders, including:

\section{Strengths and limitations of this study}

- Our review represents the first step towards the development of a standardised initiative to inform outcome selection for trials assessing the effectiveness of interventions used to treat major depressive disorder in older adults.

- Strengths of our review include: use of rigorous methodology to conduct data screening and extraction, and grouping of outcomes according to a standardised classification system.

- The generalisability of our findings may be limited by our search strategy which focuses on published, peer-reviewed, English-language trials from 2011 and onwards.

- Our ability to synthesise findings may be impeded by the heterogeneity in reported outcomes, and the subjective reporting of outcomes as either effectiveness or harms/adverse events across trials.

- Findings from this review will contribute to the development of a core outcome set for older adults with depression, with the aim of reducing variability in outcome selection, measurement and reporting across trials examining this clinical population.

major depressive disorder (MDD), bipolar disorder and cyclothymic disorder. MDD, hereafter referred to as depression, is the most common of mood disorders, and one of the leading causes of disability for older adults worldwide, accounting for $1.7 \%$ (95\% $\mathrm{CI}=1.3 \%$ to $2.3 \%$ ) of global disability-adjusted life years. ${ }^{3}$ Older adults with depression often experience adverse health outcomes, including a reduced quality of life, ${ }^{4}$ disability ${ }^{5}$ and mortality, ${ }^{6}$ in addition to placing a high burden on caregivers ${ }^{7}$ and increased costs for healthcare systems. ${ }^{8}$

Common interventions for treating MDD in older adults include but are not limited to: pharmacotherapy (antidepressants, eg, selective serotonin reuptake inhibitors, tricyclic 
antidepressants monoamine oxidase inhibitors and other forms of medication such as antipsychotics and lithium in treatment-resistant depression), ${ }^{9}$ psychotherapy (eg, cognitive-behavioural therapy, interpersonal therapy and psychodynamic therapy), ${ }^{10}{ }^{11}$ light therapy, ${ }^{12}{ }^{13}$ exercise therapy ${ }^{14}$ and neurostimulation treatments (eg, electroconvulsive therapy and repetitive transcranial simulation). ${ }^{1516}$ Randomised controlled trials (RCTs) are widely considered the gold standard for assessing comparative effectiveness of therapeutic interventions. ${ }^{17}$ These trials are essential to understanding the unique clinical profiles of this population, given that comorbid mental and physical illnesses which often accompany ageing must be considered during the selection of treatment course. For instance, the use of antidepressants, although effective in treating depressive symptomatology, may result in adverse events due to comorbidities and drug-drug interactions if patients are on concomitant medications. ${ }^{9}$ Furthermore, there is a need to consider other social factors which affect non-adherence to prescribed treatment regimens among older adults with MDD, including but not limited to: socioeconomic status and insurance coverage, educational level and health literacy. ${ }^{18}$ Unfortunately, recent meta-analyses synthesising trials for depression among older adults have exhibited high heterogeneity, both in the use of dissimilar outcomes across studies, as well as differences in instruments used to measure the same set of outcomes, ${ }^{19}{ }^{20}$ which creates challenges for the interpretation of trial results and limits their utility in clinical decision-making.

The inconsistency in the selection and reporting of outcomes for RCTs is a well-established issue in medical research, ${ }^{21-24}$ as little thought has been given to what constitutes a successful treatment outcome and measurement of outcome domains. In particular, the rationale for the use of a particular outcome and evidence to support the rationale,${ }^{25}$ are infrequently, if ever, provided in studies which evaluate effectiveness of depression interventions. ${ }^{26}$ Although it is considered that different outcomes assess the severity of depression similarly and can therefore be used interchangeably, a content analysis revealed only a moderate mean overlap (Jaccard index $=0.41 ; 0=$ no overlap, $1=$ complete overlap) between clinical rating scales, which have been commonly used as outcome measures in depression trials. ${ }^{26}{ }^{27}$ Researchers and clinicians alike have also questioned the suitability of outcome measurements used in depression trials to gauge treatment effectiveness, ${ }^{28-30}$ and this variability in outcome measurements between studies makes it difficult to first determine the effectiveness of interventions and second, poses challenges for the generalisability of research conducted on depression. ${ }^{31} 32$

Furthermore, there is a need to consider which outcomes are important to patients suffering from MDD. Prior research on patients with depression has identified that outcomes which patients consider as markers of treatment success largely pertain to improvements in functional capacity, and include domains pertaining to social life, that is, reductions in social isolation, improved quality of interpersonal relationships and family life and the ability to resume professional responsibilities. ${ }^{33}$ These patient-important outcomes (PIO) are often neglected in RCTs which evaluate treatment success from clinician and policymaker perspectives, for example, improvements in depression symptoms as measured by standardised clinical scales. Establishing a common set of treatment outcomes for depression needs to consider the diverse perspectives of all stakeholders involved. Specifically, there is a clear gap in research in identifying measurable treatment outcomes that have a significant impact on improving the lives of the patient population they are designed to treat, and including PIO as measures of effectiveness in RCTs.

Core outcome sets (COS) have been proposed as a viable solution to addressing the heterogeneity of outcome selection in RCTs, ${ }^{21}{ }^{22} 24$ and represent a minimum set of outcomes that must be measured and reported in trials pertaining to a particular illness (what to measure).$^{34} \mathrm{COS}$ are developed through systematic scans of the literature and consensus meetings to determine the outcomes used in these sets, for example, Delphi surveys and in-person meetings with clinicians, patients and policymakers. ${ }^{35}$ After a core set of outcomes has been identified, the outcome measurement instrument (how) and details of the measurement (when) are subsequently assessed through similar means, that is, literature scans and Delphi studies. ${ }^{36}$ The Core Outcome Measures in Effectiveness Trials Initiative is a repository of COS for different medical conditions. ${ }^{37}$ Although there have been initiatives towards the development of adolescent $t^{38-40}$ and general adult ${ }^{33}$ depression, there is no COS which exists for use in studies for older adults with MDD. Thus, trialists, clinicians and other evidence users lack an evidencebased means of selecting, measuring and reporting outcomes for this clinical population.

Our methodological review is the first step towards the development of a COS for RCTs evaluating treatments for older adults with depression. In this protocol, we outline the methods we will use to identify and describe outcomes reported in published trials for this clinical population. The findings from our methodological scan of the literature will be used to evaluate the extent of heterogeneity present in trials, assess whether PIO have been used in these studies, and identify a preliminary list of outcomes to consider for developing a COS for older adults with MDD.

\section{OBJECTIVES}

Our overarching goal is to identify and characterise outcomes reported in published trials of older adults with MDD. Our specific objectives are as follows:

1. To describe outcomes that have been reported in RCTs for older adults with depression (primary study objective). a. To map reported outcomes to core outcome areas/ taxonomic classifications which have been used in 
biomedical research: physiological/clinical, life impact, resource use, adverse events and death. ${ }^{41}$

2. To identify whether reported outcomes are consistent with relevant outcomes for depression which matter to patients, informal caregivers and healthcare providers $^{33}$ (secondary study objective).

3. To identify how many trials use a single discernible primary outcome and report on the rationale for their use (tertiary study objective).

Findings from our study will be used to evaluate the extent of the heterogeneity present in outcome selection, and present an initial list of outcomes to consider towards the development of a COS for this clinical population, which are aligned with PIO.

\section{METHODS AND ANALYSIS \\ Study design}

We will conduct a methodological review of outcomes reported in published RCTs of interventions used to treat MDD in older adults. This is the most appropriate type of review for our study objectives, which seek to identify and map outcomes in this trial, and has been used in other domains. ${ }^{42-44}$

\section{Protocol}

Our protocol includes elements commonly used in protocols for other methodological reviews and/or methodological surveys of trials. ${ }^{45-49}$ Any amendments to this protocol will be detailed in the final publication. Our review will commence after peer review of this paper, and is anticipated to be completed by December 2021. This methodological review has been registered on PROSPERO.

\section{Eligibility criteria}

Studies included in our review will satisfy the following eligibility criteria, which have been based on the Population, Intervention, Comparators, Outcomes, Time, and Studies (PICOTS) framework. ${ }^{50-52}$

\section{Population (P)}

Older adults aged 65 years and older with a diagnosis of MDD, as defined by the Diagnostic and Statistical Manual of Mental Disorders (4th or 5th editions) ${ }^{53}$ or depressive disorder as defined by the International Statistical Classification of Diseases (10th edition) criteria. ${ }^{54}$ This age cut-off is a common threshold used in geriatric psychiatry. ${ }^{95}$ Diagnoses for depression will include a diagnosis by a psychiatrist, through administrative health records or clinical charts, or by use of a validated diagnostic tool, including but not limited to: the Beck Depression Inventory (BDI) ${ }^{56}$ Hamilton Rating Scale for Depression ${ }^{57}$ or the Center of Epidemiological Scale (CES-D) ${ }^{58}$ We will focus our review on unipolar and non-psychotic MDD, given that intervention choices may differ for these aforementioned forms of depression. Studies of patients with comorbid mental disorders, for example, anxiety and depression or schizophrenia and depression will be considered for inclusion, as comorbid mental illness is common among older adults. ${ }^{59} 60$ RCTs which include participants with different ages will be included only if there is a subgroup analysis containing adults aged 65 and older (eg, trials analysing a subgroup of adults aged $70-80$ years would be eligible, but a subgroup analysis of ages 55-70 would not be eligible).

\section{Intervention (I)}

All interventions (pharmacological and nonpharmacological) will be considered for inclusion.

\section{Comparators (C)}

There will be no restrictions or specifications regarding comparators.

\section{Outcomes (0)}

All outcomes specified in published studies of randomised group comparisons will be eligible for inclusion. These may include outcomes pertaining to: delivery of care (eg, treatment adherence, intervention acceptability to patients), health status (eg, severity of depression) and/ or health service use (eg, numbers of psychiatric appointments, emergency department visits or general practitioner visits). ${ }^{41}$ All outcomes will be collected in order to provide a comprehensive assessment of outcomes in trials of older adults with MDD and in accordance with recommendations followed by accepted taxonomy towards development of a $\mathrm{COS}^{41}$ We will not include adverse events that emerge as a result of treatment and are detected through site visits, for example, headaches, as these are not planned outcomes of interest to assess the effectiveness of interventions and are pertinent to an intervention in a particular study.

\section{Time (T)}

Studies published within the last 10 years will be considered for inclusion in our review, so that we are reporting on trials which have been recently conducted. This approach is consistent with other methodological reviews. ${ }^{38-40} 45464849$ Furthermore, COS development guidelines suggest that overly large reviews are not necessary to yield additional outcomes, and that shorter timeframes for reviews may suffice in reaching saturation of outcome terminology. ${ }^{35}$ There will be no restrictions imposed on timing of outcome measurement, for example, when they have been measured post-enrollment, or on duration of follow-up post administration of the intervention.

\section{Studies (S)}

English-language RCTs will be eligible for inclusion, the language restriction in place for feasibility considerations. We will consider trials which have been conducted in all countries and settings (eg, inpatient, outpatient and community) in our review. We will not impose restrictions on RCT design, that is, single-arm, parallel, multi-arm and crossover trials will be eligible for inclusion. We will 


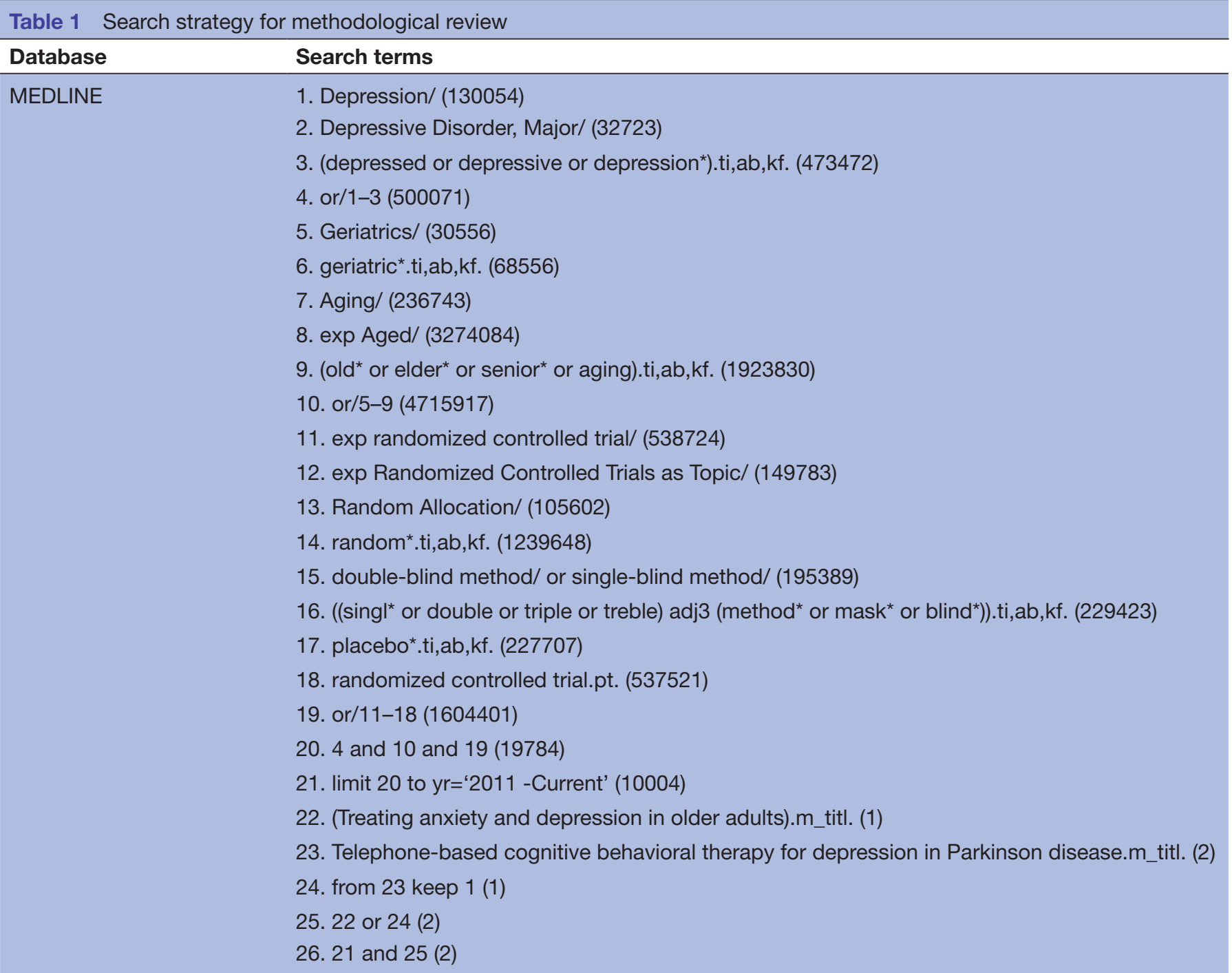

also include pilot and feasibility trials only when the fullscale RCT is not available for inclusion, so as to prevent duplication of reported outcomes.

\section{Information sources and search strategy}

Our search strategy and information sources have been developed in consultation with a health sciences librarian (SS), and will include the following databases: MEDLINE, Embase, PsycINFO and the Cochrane Central Register of Controlled Trials. Comprehensive search strategies (see table 1) will be used in the above databases to obtain articles using searches from 1 January 2011 until the date of the conducted search. Our search strategy has been developed through analysis of Medical Subject Headings terms and keywords identified though a preliminary search of the literature for relevant publications. Table 1 illustrates terms used in the most encompassing database, however final search strategies for all databases will be included in the review. De-duplication of records will be performed in the Covidence software platform. ${ }^{61}$

We will also conduct manual searches of reference lists of all included studies to identify potentially missed trials. Articles identified from the reference lists will be reviewed in duplicate (detailed below) and included if they meet the aforementioned eligibility criteria. Conference reports, abstracts, unpublished reports or trial registries and other forms of literature that have not been peer-reviewed will not be included, because our objectives are to assess the measurement of outcomes in published trials.

\section{Data management}

We will import articles identified through the search strategies to the Covidence software platform, in order to conduct all phases of the review process. Covidence has the ability to store citation information imported from reference management software, and will be used to manage (1) title and abstract and (2) full-text screening, as well as (3) data extraction of included studies. Covidence permits study team members to review citation information independently and in duplicate, and extract data directly into forms on the software platform. $^{61}$ 


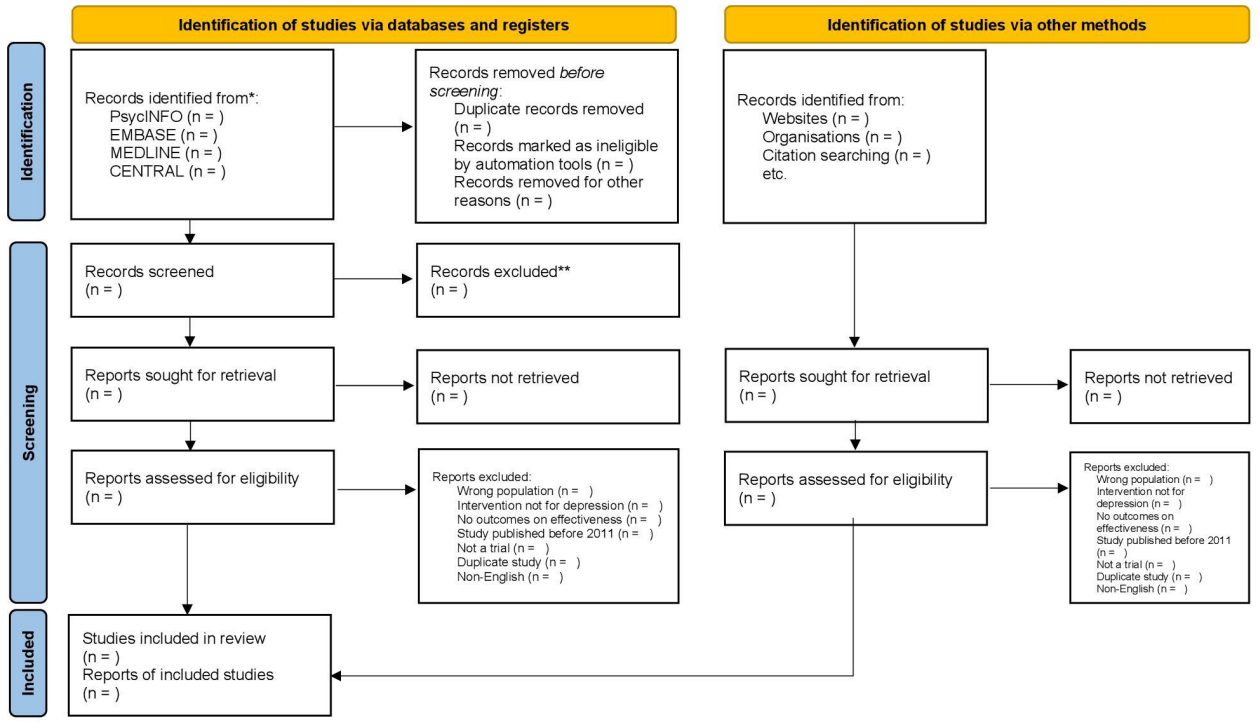

Figure 1 Flow diagram of included studies.

\section{Source selection}

We will include a flow diagram (see figure 1) to summarise the screening process in our systematic reviews and meta-analyses. This will be structured according to the Preferred Reporting Items for Systematic Reviews and Meta-Analyses (PRISMA) - 2020 guidelines ${ }^{62}$ and detail numbers of studies and reasons for inclusion/exclusion. Included studies will also be described and detailed in a table.

\section{Title and abstract screening}

We will conduct title and abstract screening of every citation identified by the literature searches to assess eligibility of studies for inclusion in our review. Two reviewers will screen each citation independently and duplicate using the aforementioned PICOTS eligibility criteria at the title and abstract stage. Study team members will be instructed to err towards inclusion, that is, include studies if they are unsure about whether or not it meets eligibility criteria. Disagreements about study inclusion between reviewers will be resolved by a third reviewer to reach consensus. We will also assess level of agreement between study reviewers using the weighted kappa $(\kappa)$ statistic $^{63}$ calculated by Covidence. Studies will move on to the full-text screening stage if they have been selected for inclusion by both reviewers or have unresolved discrepant decisions regarding inclusion eligibility.

\section{Full-text screening}

During the full-text screening stage, two reviewers will screen each full-text article independently and duplicate. Disagreements about study inclusion between reviewers will be resolved by a third reviewer to reach consensus. We will contact authors, if necessary, if there is confusion regarding eligibility criteria. The level of agreement between study reviewers will be assessed at this stage using the weighted $\mathrm{\kappa}$ statistic ${ }^{63}$ calculated by Covidence. Reasons for study exclusion will be logged used Covidence and detailed in the PRISMA study flow diagram (figure 1). Included studies will move to the next stage into data extraction.

If there are multiple publications from a single trial, for example, several studies using the same patient population, we will select a single trial for inclusion in our review. In order to prevent duplicate study entries, we will select a trial: (1) with the largest sample size, (2) that has been published most recently, in case the sample sizes are the same across publications, (3) has the most number of outcomes, in case the sample size and publication years are the same or (4) applying a combination of the above criteria for inclusion. Decisions about study inclusion for duplicate trials will also be made independently and by two reviewers, with disagreements resolved by a third reviewer as needed.

\section{Data extraction}

Two members of the study team will perform data extraction independently and in duplicate for every included unique trial. After data charting has been independently completed by two reviewers, they will resolve disagreements through consensus, and with a third reviewer as needed. A standardised charting form will be developed a priori (see Online Supplementary Appendix A), and we will extract data pertaining to study and outcome characteristics. We will extract study-specific data pertaining to: study identifiers (eg, lead author surname, year of publication, name of journal), trial design (eg, single-arm, parallel, multi-arm or crossover), trial type (eg, full-scale, pilot or feasibility), study characteristics (eg, total sample size, intervention type-pharmacological or non-pharmacological, mean participant age, follow-up duration, study setting region and source of funding-industry-funded or not).

We will also extract the following information pertaining to outcomes: definition of the outcome or endpoint 
used in the trial, definition of meaningful change specified in the study, type of outcome measure (eg, single or composite outcome) and instruments used to measure outcome(s) (eg, BDI, CES-D or other standardised clinical scales). For instance, trials of older adults with depression often use the BDI (measurement instrument) to assess severity of depressive symptomatology (outcome). We will also extract whether study outcomes have been classified as: (1) primary, (2) secondary or (3) neither. The 'primary' outcome classification will apply to an outcome which: (1) studies have explicitly referred to using this language, (2) have been clearly specified in the objectives, that is, when studies aim to examine the effect of an intervention on a particular outcome or (3) in instances where the outcome data has been used to power the sample size for the trial. ${ }^{3964}$ Given that multiple primary outcomes are often reported in trials of depression, ${ }^{65}$ we wish to capture the extent of this occurrence in our methodological review.

For studies which report a single, discernable primary outcome, we will extract: the source of information of the outcome (who), description of the outcome (what), location of assessment/setting of the outcome (where), timing of outcome measurement (when), rationale for selection of the outcome (why) and description of the means by which the outcome has been measured (how). We will also extract how outcome data has been managed and analysed, in addition to strategies employed in the analysis and interpretation of missing data. ${ }^{38} 39$

\section{Data synthesis}

Grouping of outcomes

After data extraction, outcomes will be grouped and synthesised using a thematic framework. This approach has been commonly used in other reviews and COS initiatives. $^{38-40} 45-49646667$ For instance, we will use the term 'social functioning' to group outcomes relating to selfreported improvement in marital/spousal relationships and 'delivery of care' for patient satisfaction and treatment adherence. We will group individual components of composite outcomes under the appropriate terms. All outcome terms will be assigned to an outcome domain framework in consultation with psychiatrists (AD and ZS) and methodological experts (LT and SP).

Outcomes will be assigned or mapped according to two existing frameworks, the first of which has been used frequently in biomedical research. We will first assign all outcome terms to the taxonomic classification system proposed by Dodd and colleagues ${ }^{41}$, which comprise five domains: physiological/clinical, life impact, resource use, adverse events and death. ${ }^{41}$ We will also map outcome terms to outcomes which were mapped by a recent content analysis and identified outcomes for depression which matter to patients, informal caregivers and healthcare providers, including: symptoms, functioning, intervention safety, healthcare organisation and social representation. ${ }^{33}$

\section{Synthesis of results}

Our data synthesis of included studies will largely be descriptive. We will include quantitative measures (eg, counts, frequencies) of study characteristics (eg, the number of single-arm, parallel, multi-arm and crossover trials) and outcome-related information (eg, total number of outcomes per trial, total number of primary, secondary and other types of outcomes, median number of outcomes per study and the total number of outcome measurement instruments per trial). Tables will be used to display the characteristics of included studies, as well as outcome characteristics (ie, types and measurement), in addition to detailing the variation in outcome definitions present across the included trials. We will also report whether outcomes reported in trials that focus on older adults specifically, are different from those reported by trials which focus on other age groups, but include subgroup analyses of older populations.

We will map outcome terms to the two aforementioned frameworks using a modified outcome matrix which was developed by the Outcome Reporting for Brief Intervention Trials initiative ${ }^{68}$ and adopted for use by other COS reviews. ${ }^{39} 40646667$

\section{Pilot testing}

The lead author (MR) will conduct online tutorials to train study team members on use of the Covidence software. We will also conduct a calibration exercise, and pilot the full-text screening and data extraction of 10 articles by each research team member for response accuracy and adherence to the protocol and inclusion/exclusion criteria before the full-text screening and data extraction stages commence.

\section{Risk of bias assessment or quality appraisal}

We will not conduct risk of bias assessment or quality appraisal of included studies, since our aim is to report on outcome measurements. This approach is consistent with that employed by other methodological reviews. ${ }^{38-40} 45-49$

\section{Patient and public involvement}

Given that this is a methodological review, there was no patient or public involvement in its conception, and they will not be involved in its conduct, either.

\section{DISCUSSION}

\section{Strengths and limitations}

Our methodological review is the first of its kind to our knowledge that aims to systematically assess reported treatment outcomes for MDD in trials of older adults. Our review will follow rigorous methodology, including a search strategy developed in consultation with an experienced librarian and duplicate screening and data extraction. We will also use the expertise of both psychiatrists and methodologists in grouping and mapping outcome terms, in accordance with a standardised classification system. ${ }^{41}$ 
However, our review is not without limitations. First, our search strategy is limited to RCTs published in the English language from 2011 onwards, and does not include abstracts, reports or trial registries. Although this restriction is aligned with our objectives and commonly practiced by other methodological reviews, it may nonetheless reduce the generalisability of our findings. Furthermore, prior research suggests that outcome reporting in RCTs not published in English may also be heterogeneous, ${ }^{69}$ about which our review cannot infer. Second, use of the COS taxonomy may not be ideal to classify all outcomes in reported trials. For instance, a methodological review on adolescent depression RCTs found that all outcomes were easily classified into the core domains suggested by the COS framework. ${ }^{38}{ }^{40}$ In particular, within the context of depression, outcomes may be subjectively reported as either effectiveness or harms/adverse outcomes, further contributing to challenges in interpreting and synthesising trial results in our review.

\section{Implications}

The conduct of high-quality RCTs is crucial for the measurement and definition of outcomes which are aligned with knowledge synthesis approaches to gauge effectiveness of interventions among older adults with MDD. Both intervention effectiveness as well as generalisability of research are limited when choice of outcome measures are arbitrary, ${ }^{31}{ }^{32}$ yet research on older adults with depression has rarely given thoughtful consideration to selection of an outcome measure, as very few studies provide the rationale and/or evidence to support their choice of a treatment outcome. ${ }^{25}{ }^{26}$ Furthermore, PIO elicit insight on treatment effectiveness from patient perspectives and are critical to informed decision-making, but have been largely ignored in health research.

Our methodological review will identify the heterogeneity of outcomes in published trials for older adults with depression, and conclusions from our review will have implications for a core set of treatment outcomes used in studies which assess effectiveness of interventions for depression, including incorporation of patient perspectives on outcomes valued as markers of treatment success. Our findings will influence recommendations on future research conducted among people with depression, with respect to possible gaps and deficiencies in selection, rationality and reporting of treatment outcomes. Our review will also contribute towards the development of a COS used in trials for older adults with depression, with the aim of improving and standardising outcome selection. Furthermore, our results will also be of interest to a diverse group of stakeholders, including authors of methodological and systematic reviews, developers of clinical care guidelines, funding agencies and decision-makers in healthcare, who rely on information regarding treatment outcomes to assess effectiveness of various interventions in treating older adults with depression.

\section{Ethics and dissemination}

We do not require ethics approval for this study. The results from our methodological review will be disseminated in peer-reviewed journals and conference presentations to knowledge users, clinicians and other researchers.

\section{Author affiliations}

${ }^{1}$ Health Research Methodology Graduate Program, Department of Health Research Methods, Evidence and Impact, McMaster University, Hamilton, Ontario, Canada

${ }^{2}$ Department of Psychiatry and Behavioral Neurosciences, McMaster University, Hamilton, Ontario, Canada

${ }^{3}$ Health Sciences Library, McMaster University, Hamilton, Ontario, Canada ${ }^{4}$ Neuroscience Graduate Program, McMaster University, Hamilton, Ontario, Canada ${ }^{5}$ Department of Health Research Methods, Evidence and Impact, McMaster University, Hamilton, Ontario, Canada

${ }^{6}$ Department of Oncology, McMaster University, Hamilton, Ontario, Canada ${ }^{7}$ Mood Disorders Program, St Joseph's Healthcare Hamilton, Hamilton, Ontario, Canada

${ }^{8}$ Population Health Research Institute, Hamilton, Ontario, Canada

\section{Twitter Myanca Rodrigues @MyancaRodrigues}

Contributors MR: contributed to the conception and design of the study and study protocol, the writing and final review of the manuscript, and assisted in the development of the search strategy and data collection tool. SS: contributed critically to the development of the search strategy and final review of the manuscript. NS, AD, BP, AD'E and SP: provided critical revision and review of the final manuscript. ZS and LT: contributed to the conception and design of the study, and provided critical revision and approval of the final manuscript. All authors read and approved the final manuscript.

Funding This work is not funded by a specific grant. ZS received funding from Alternate Funding Plan (No. 20-10178-480925-75153), and Canadian Institutes for Health Research (Award No. PJT-156306).

Competing interests None declared.

Patient consent for publication Not applicable.

Provenance and peer review Not commissioned; externally peer reviewed.

Supplemental material This content has been supplied by the author(s). It has not been vetted by BMJ Publishing Group Limited (BMJ) and may not have been peer-reviewed. Any opinions or recommendations discussed are solely those of the author(s) and are not endorsed by BMJ. BMJ disclaims all liability and responsibility arising from any reliance placed on the content. Where the content includes any translated material, BMJ does not warrant the accuracy and reliability of the translations (including but not limited to local regulations, clinical guidelines, terminology, drug names and drug dosages), and is not responsible for any error and/or omissions arising from translation and adaptation or otherwise.

Open access This is an open access article distributed in accordance with the Creative Commons Attribution Non Commercial (CC BY-NC 4.0) license, which permits others to distribute, remix, adapt, build upon this work non-commercially, and license their derivative works on different terms, provided the original work is properly cited, appropriate credit is given, any changes made indicated, and the use is non-commercial. See: http://creativecommons.org/licenses/by-nc/4.0/.

\section{ORCID iDs}

Myanca Rodrigues http://orcid.org/0000-0001-7953-773X

Nitika Sanger http://orcid.org/0000-0002-5883-1873

Balpreet Panesar http://orcid.org/0000-0002-5492-3615

Alessia D'Elia http://orcid.org/0000-0002-6512-4704

Zainab Samaan http://orcid.org/0000-0002-5974-9361

Lehana Thabane http://orcid.org/0000-0003-0355-9734

\section{REFERENCES}

1 McRae L, O'Donnell S, Loukine L. Report summary - mood and anxiety disorders in Canada, 2016 TT - Note de synthèse - Les troubles anxieux et de l'humeur au Canada, 2016. Heal Promot chronic Dis Prev Canada Res policy Pract 2016;36:314-5.

2 Watson D. Rethinking the mood and anxiety disorders: a quantitative hierarchical model for DSM-V. J Abnorm Psychol 2005;114:522-36. 
3 GBD 2019 Diseases and Injuries Collaborators. Global burden of 369 diseases and injuries in 204 countries and territories, 1990-2019: a systematic analysis for the global burden of disease study 2019 . Lancet 2020;396:1204-22.

4 Fassino S, Leombruni P, Abbate Daga G, Daga GA, et al. Quality of life in dependent older adults living at home. Arch Gerontol Geriatr 2002;35:9-20.

5 Beekman ATF, Penninx BWJH, Deeg DJH, et al. The impact of depression on the well-being, disability and use of services in older adults: a longitudinal perspective. Acta Psychiatr Scand 2002;105:20-7.

6 Abas M, Hotopf M, Prince M. Depression and mortality in a high-risk population. 11-year follow-up of the medical Research Council elderly hypertension trial. Br J Psychiatry 2002;181:123-8.

7 Baldwin RC, Anderson D, Black S, et al. Guideline for the management of late-life depression in primary care. Int $J$ Geriatr Psychiatry 2003;18:829-38.

8 Gustavsson A, Svensson M, Jacobi F, et al. Cost of disorders of the brain in Europe 2010. Eur Neuropsychopharmacol 2011;21:718-79.

9 Kok RM, Reynolds CF. Management of depression in older adults: a review. JAMA 2017;317:2114-22.

10 Jayasekara R, Procter N, Harrison J, et al. Cognitive behavioural therapy for older adults with depression: a review. J Ment Health 2015;24:168-71.

11 Wilson KCM, Mottram PG, Vassilas CA. Psychotherapeutic treatments for older depressed people. Cochrane Database Syst Rev 2008:CD004853.

12 Perera S, Eisen R, Bhatt M, et al. Light therapy for non-seasonal depression: systematic review and meta-analysis. BJPsych Open 2016;2:116-26.

13 Zhao X, Ma J, Wu S, et al. Light therapy for older patients with nonseasonal depression: a systematic review and meta-analysis. J Affect Disord 2018;232:291-9.

14 Schuch FB, Vancampfort D, Rosenbaum S, et al. Exercise for depression in older adults: a meta-analysis of randomized controlled trials adjusting for publication bias. Braz $J$ Psychiatry 2016;38:247-54.

15 Geduldig ET, Kellner $\mathrm{CH}$. Electroconvulsive therapy in the elderly: new findings in geriatric depression. Curr Psychiatry Rep 2016;18:40.

16 Rabheru K. The use of electroconvulsive therapy in special patient populations. Can J Psychiatry 2001;46:710-9.

17 Hariton E, Locascio JJ. Randomised controlled trials - the gold standard for effectiveness research. BJOG an Int J Obstet Gynaecol 1716;2018:125.

18 MacLaughlin EJ, Raehl CL, Treadway AK, et al. Assessing medication adherence in the elderly. Drugs Aging 2005;22:231-55.

19 Kok RM, Nolen WA, Heeren TJ. Efficacy of treatment in older depressed patients: a systematic review and meta-analysis of double-blind randomized controlled trials with antidepressants. $J$ Affect Disord 2012;141:103-15.

20 Mallery L, MacLeod T, Allen M, et al. Systematic review and meta-analysis of second-generation antidepressants for the treatment of older adults with depression: questionable benefit and considerations for frailty. BMC Geriatr 2019;19:306.

21 Gorst SL, Gargon E, Clarke M, et al. Choosing important health outcomes for comparative effectiveness research: an updated review and user survey. PLoS One 2016;11:e0146444.

22 Williamson P, Altman D, Blazeby J, et al. Driving up the quality and relevance of research through the use of agreed core outcomes. $J$ Health Serv Res Policy 2012;17:1-2.

23 Macleod MR, Michie S, Roberts I, et al. Biomedical research: increasing value, reducing waste. Lancet 2014;383:101-4.

24 Idzerda L, Rader T, Tugwell P, et al. Can we decide which outcomes should be measured in every clinical trial? A scoping review of the existing conceptual frameworks and processes to develop core outcome sets. J Rheumatol 2014;41:986 LP - 993

25 Gidron Y. Reliability and validity. In: Gellman MD, Turner JR, eds. Encyclopedia of behavioral medicine. New York, NY: Springer New York, 2013: 1643-4.

26 Fried El. The 52 symptoms of major depression: lack of content overlap among seven common depression scales. J Affect Disord 2017:208:191-7.

27 Fried El. Corrigendum to "The 52 symptoms of major depression: lack of content overlap among seven common depression scales", [Journal of Affective Disorders, 208, 191-197]. J Affect Disord 2020;260:744.

28 Zimmerman M, Martinez JH, Friedman M, et al. How can we use depression severity to guide treatment selection when measures of depression categorize patients differently? J Clin Psychiatry 2012;73:1287-91.
29 Kearns NP, Cruickshank CA, McGuigan KJ, et al. A comparison of depression rating scales. Br J Psychiatry 1982;141:45-9.

30 Cusin C, Yang H, Yeung A. Rating scales for depression. In: Handbook of clinical rating scales and assessment in psychiatry and mental health. New York: Humana Press, 2010: 7-35.

31 Santor DA, Gregus M, Welch A. Focus article: eight decades of measurement in depression. Meas Interdiscip Res Perspect 2006;4:135-55

32 Snaith P. What do depression rating scales measure? Br J Psychiatry 1993;163:293-8.

33 Chevance A, Ravaud P, Tomlinson A, et al. Identifying outcomes for depression that matter to patients, informal caregivers, and healthcare professionals: qualitative content analysis of a large international online survey. Lancet Psychiatry 2020;7:692-702.

34 Williamson PR, Altman DG, Blazeby JM, et al. The comet (core outcome measures in effectiveness trials) initiative. Trials 2011;12:A70

35 Williamson PR, Altman DG, Bagley $\mathrm{H}$, et al. The comet Handbook: version 1.0. Trials 2017;18:280.

36 Prinsen CAC, Vohra S, Rose MR, et al. How to select outcome measurement instruments for outcomes included in a "Core Outcome Set" - a practical guideline. Trials 2016;17:449.

37 COMET Initiative. Core outcome measures in effectiveness trials, 2021. Available: https://www.comet-initiative.org/

38 Monsour A, Mew EJ, Patel S, et al. Primary outcome reporting in adolescent depression clinical trials needs standardization. BMC Med Res Methodol 2020;20:129.

39 Monsour A, Mew EJ, Szatmari P, et al. Outcomes reported in randomised clinical trials of major depressive disorder treatments in adolescents: a systematic scoping review protocol. BMJ Open 2019:9:e024191.

40 Mew EJ, Monsour A, Saeed L, et al. Systematic scoping review identifies heterogeneity in outcomes measured in adolescent depression clinical trials. J Clin Epidemiol 2020;126:71-9.

41 Dodd S, Clarke M, Becker L, et al. A taxonomy has been developed for outcomes in medical research to help improve knowledge discovery. J Clin Epidemiol 2018;96:84-92.

42 Chiarotto A, Ostelo RW, Turk DC, et al. Core outcome sets for research and clinical practice. Brazilian J Phys Ther 2017;21:77-84.

43 Boers M, Brooks P, Strand CV, et al. The OMERACT filter for outcome measures in rheumatology. J Rheumatol 1998;25:198-9.

44 Felson DT, Anderson JJ, Boers M, et al. The American College of rheumatology preliminary core set of disease activity measures for rheumatoid arthritis clinical trials. The Committee on outcome measures in rheumatoid arthritis clinical trials. Arthritis Rheum 1993;36:729-40.

45 Scola LFC, Moseley AM, Thabane L, et al. A methodological survey on reporting of pilot and feasibility trials for physiotherapy interventions: a study protocol. BMJ Open 2019;9:e020580.

46 Madden K, Arseneau E, Evaniew N, et al. Reporting of planned statistical methods in published surgical randomised trial protocols: a protocol for a methodological systematic review. BMJ Open 2016;6:e011188.

47 Clarke M, Oxman AD, Paulsen E. Appendix A: guide to the contents of a Cochrane methodology protocol and review. Cochrane Handb Syst Rev Interv 2011.

48 Lawson DO, Leenus A, Mbuagbaw L. Mapping the nomenclature, methodology, and reporting of studies that review methods: a pilo methodological review. Pilot Feasibility Stud 2020;6:13.

49 Devji T, Johnston BC, Patrick DL, et al. Presentation approaches for enhancing interpretability of patient-reported outcomes (pros) in meta-analysis: a protocol for a systematic survey of Cochrane reviews. BMJ Open 2017;7:e017138.

50 Thabane L, Thomas T, Ye C, et al. Posing the research question: not so simple. Can J Anesth/J Can Anesth 2009;56:71-9.

51 Sackett DL, Haynes RB, Guyatt GH. Clinical epidemiology: how to do clinical practice research 2005

52 Richardson WS, Wilson MC, Nishikawa J, et al. The well-built clinical question: a key to evidence-based decisions. ACP J Club 1995;123:A12-13.

53 American Psychiatric Association. Diagnostic and statistical manual of mental disorders (DSM-5®). American Psychiatric Association, 2013

54 World Health Organization. International statistical classification of diseases and related health problems: Tabular list. Geneva: World Health Organization, 2004

55 Rodda J, Walker Z, Carter J. Depression in older adults. BMJ 2011;343:d5219.

56 Beck AT, Ward CH, Mendelson M, et al. An inventory for measuring depression. Arch Gen Psychiatry 1961;4:561-71. 
57 Hamilton M. A rating scale for depression. J Neurol Neurosurg Psychiatry 1960;23:56-62.

58 Radloff LS. The CES-D scale: a self-report depression scale for research in the general population. Appl Psychol Meas 1977;1:385-401.

59 Dai J, Du X, Yin G, et al. Prevalence, demographic and clinical features of comorbid depressive symptoms in drug naïve patients with schizophrenia presenting with first episode psychosis. Schizophr Res 2018;193:182-7.

60 Hek K, Tiemeier H, Newson RS, et al. Anxiety disorders and comorbid depression in community dwelling older adults. Int $J$ Methods Psychiatr Res 2011;20:157-68.

61 Covidence. Covidence systematic review software [Computer Software] 2017.

62 Page MJ, McKenzie JE, Bossuyt PM, et al. The PRISMA 2020 statement: an updated guideline for reporting systematic reviews. BMJ 2021;372:n71.

63 McHugh ML. Interrater reliability: the kappa statistic. Biochem Med 2012;22:276-82.
64 Hall NJ, Kapadia MZ, Eaton S, et al. Outcome reporting in randomised controlled trials and meta-analyses of appendicitis treatments in children: a systematic review. Trials 2015;16:1-14.

65 Tyler KM, Normand S-LT, Horton NJ. The use and abuse of multiple outcomes in randomized controlled depression trials. Contemp Clin Trials 2011;32:299-304.

66 Shorter GW, Bray JW, Giles EL, et al. The variability of outcomes used in efficacy and effectiveness trials of alcohol brief interventions: a systematic review. J Stud Alcohol Drugs 2019;80:286-98.

67 Shorter GW, Heather N, Bray JW, et al. The 'Outcome reporting in brief intervention trials: Alcohol' (ORBITAL) framework: protocol to determine a core outcome set for efficacy and effectiveness trials of alcohol screening and brief intervention. Trials 2017;18:1-7.

68 Kirkham JJ, Dwan KM, Altman DG, et al. The impact of outcome reporting bias in randomised controlled trials on a cohort of systematic reviews. BMJ 2010;340:c365.

69 Turner L, Shamseer L, Altman DG, et al. Consolidated standards of reporting trials (consort) and the completeness of reporting of randomised controlled trials (RCTs) published in medical journals. Cochrane Database Syst Rev 2012;11:MR000030. 